\title{
Functional metagenomic characterization of antibiotic resistance genes in agricultural soils from China
}

\author{
Jian Qiang Su ${ }^{\mathrm{a}}$, Bei Wei ${ }^{\mathrm{a}}$, Chun Yan Xu ${ }^{\mathrm{a}}$, Min Qiao ${ }^{\mathrm{b}}$, Yong Guan Zhu ${ }^{\mathrm{a}, \mathrm{b}, *}$ \\ a Key Lab of Urban Environment and Health, Institute of Urban Environment, Chinese Academy of Sciences, Xiamen 361021, China \\ b State Key Lab of Urban and Regional Ecology, Research Center for Eco-environmental Sciences, Chinese Academy of Sciences, Beijing 100085, China
}

\section{A R T I C L E I N F O}

Article history:

Received 4 June 2013

Accepted 13 December 2013

Available online 8 January 2014

\section{Keywords:}

Antibiotic resistance

Functional metagenomics

Agricultural soils

\begin{abstract}
A B S T R A C T
Soil has been regarded as a rich source of antibiotic resistance genes (ARGs) due to the complex microbial community and diverse antibiotic-producing microbes in soil, however, little is known about the ARGs in unculturable bacteria. To investigate the diversity and distribution of ARGs in soil and assess the impact of agricultural practice on the ARGs, we screened soil metagenomic library constructed using DNA from four different agricultural soil for ARGs. We identified 45 clones conferring resistance to minocycline, tetracycline, streptomycin, gentamicin, kanamycin, amikacin, chloramphenicol and rifampicin. The similarity of identified ARGs with the closest protein in GenBank ranged from $26 \%$ to $92 \%$, with more than $60 \%$ of identified ARGs had low similarity less than $60 \%$ at amino acid level. The identified ARGs include aminoglycoside acetyltransferase, aminoglycoside 6 -adenyltransferase, ADP-ribosyl transferase, ribosome protection protein, transporters and other antibiotic resistant determinants. The identified ARGs from the soil with manure application account for approximately $70 \%$ of the total ARGs in this study, implying that manure amendment may increase the diversity of antibiotic resistance genes in soil bacteria. These results suggest that antibiotic resistance in soil remains unexplored and functional metagenomic approach is powerful in discovering novel ARGs and resistant mechanisms.
\end{abstract}

() 2013 Elsevier Ltd. All rights reserved.

\section{Introduction}

Environmental contamination with antibiotics and antibiotic resistance genes (ARGs) is becoming a global health problem (Bush et al., 2011). The successful treatment for infections using antibiotics is being threatened by antibiotic resistance, which has developed from resistance to one class of antibiotics to increasingly prevalent multidrug resistance and extreme drug resistance. Antibiotic resistance has been one of the most important sustained driving forces for antibiotic discovery. Most of the research on the risk of ARGs to human health focused on the clinical setting, however, ARGs from pathogens comprise only a tiny fraction of the resistome (Wright, 2007). Soil has been regarded as a rich source of ARGs, deriving from both natural and anthropogenic processes. Although high frequency of antibiotic resistance was revealed in soils by culture-dependent approaches, it represents only a fraction of soildwelling bacteria (D'Costa et al., 2006), and uncultured soil bacteria represent a reservoir of new ARGs, which can be transferred to clinical pathogens via mobile genetic elements (Riesenfeld et al., 2004).

Culture-independent molecular approaches have provided powerful means to explore ARGs in nature and address the dissemination of ARGs in different environments. Quantitative PCR (qPCR) based approaches,

\footnotetext{
* Corresponding author at: Key Lab of Urban Environment and Health, Institute of Urban Environment, Chinese Academy of Sciences, Xiamen 361021, China. Tel.: + 86 592 6190997; fax: + 865926190514.

E-mail address: ygzhu@iue.ac.cn (Y.G. Zhu).
}

including high-throughput qPCR (Looft et al., 2012), has been applied to the quantification of ARGs for comparison of their distribution in soil, water, sediment and sewage, and also for the assessment of impacts of anthropogenic activity on the ecology of ARGs (Gaze et al., 2011; Luo et al., 2010; Popowska et al., 2012; Zhang and Zhang, 2011). Although qPCR is sensitive in detection of ARGs, it relies on specific primers designed from known target sequences, thus it cannot detect unknown ARGs. Metagenomics approach has been used to characterize ARGs in humans and environmental microbial communities and has shed new insights into the prevalence and diversity of ARGs (Monier et al., 2011). High throughput sequencing of metagenomic DNA using next generation sequencing technology has led to impressively accelerated accumulation of environmental metagenomic sequences, which provide information of the prevalence of species of interest, ARGs and mobile genetic elements in various environments (Monier et al., 2011), and enable discovery of novel ARGs. However, activity-based functional screening of metagenomic libraries, which provide direct evidence for antibiotic resistant phenotype, still remains a critical means to discover novel ARGs and identify their function that could be missed when using sequence based analysis only. Using functional screening of metagenomic libraries, novel functional ARGs have been identified from human microbiome (Sommer et al., 2009), organic pig gut (Kazimierczak et al., 2009), activated sludge (Mori et al., 2008) and soils (Allen et al., 2009; Lang et al., 2010; McGarvey et al., 2012). Most of these ARGs have not been identified previously and are evolutionarily distant from known resistance genes. 
Numerous reports describe that agricultural practices, such as direct application of antibiotics or animal manure, provide positive selective pressure for antibiotic resistant bacteria, resulting in increase number and resistant level of these bacteria and ARGs in agricultural soils, and thereby expand the level of native resistance in soil (Knapp et al., 2010; Popowska et al., 2012. In addition, a considerable amount of resistant bacteria added into soil through manure amendment would lead to the spread of resistance to soil bacteria (Ghosh and LaPara, 2007; Heuer et al., 2011). However, there are only a few studies focusing on identification and characterization of ARGs in agricultural soil using functional metagenomics. Using this method, a total of 8 new ARGs were identified from a Spanish agricultural field, conferring resistance to ampicillin, gentamicin, chloramphenicol and trimethoprim (Torres-Cortes et al., 2011). From an apple orchard soil, 13 ARGs were identified including two putative novel bi-functional protein encoding genes (Donato et al., 2010).

Recent studies have been carried out to quantify the ARGs from soil, water and sediment samples in China. In a study conducted in Haihe River, Northern China, Luo et al. revealed that sulfonamide resistance genes, sul1 and sul2, were prevalent in this river, and that the gene abundance in sediments is 120-2000 times higher than in water, suggesting that sediments are an important reservoir of ARG in Haihe River (Luo et al., 2010). In a recent survey of tetracycline resistance genes in soils adjacent to representative swine feedlots in China, 15 tetracycline resistance (tet) genes were commonly detected in soil samples and the absolute number of tet gene copies was strongly correlated with the concentrations of tetracycline residues in these soil samples $(\mathrm{Wu}$ et al., 2010).

Despite the widely recognized potential health risks of over- or misuse of antibiotics in human and animal industries in China, information on abundance and diversity of ARGs is still scarce. Therefore the aim of this study is to investigate ARGs conferring resistance to one of thirteen antibiotics and discover novel ARGs in agricultural soils using functional metagenomic screening. By using three different type of soil, we also aim to assess the impact of agricultural practices, such as manure amendment and application of antibiotics, on the prevalence and diversity of ARGs in agricultural soils.

\section{Material and methods}

\subsection{Soil samples}

Four soil samples were collected from different agricultural soils, including 1) one from a field soil (DC soil) grown with Allium fistulosum L with a history of manure amendment located at Tongzhou, Beijing, Northern China. 2) Two from paddy fields in Tianjin (QG and WG soils), northern China, without application of manure and organic compost. 3) One from shrimp pond sediment (YZC soil, previously paddy soil) at Zhangzhou, Southern China. For each sample, three $500 \mathrm{~g}$ subsamples of soil were taken from $0-10 \mathrm{~cm}$ depth and were mixed in situ to form a combined sample. Soil samples were stored in icebox for transport to laboratory and approximately $100 \mathrm{~g}$ subsamples were stored at $-80{ }^{\circ} \mathrm{C}$ for DNA extraction.

\subsection{Metagenomic library construction}

High molecular weight community DNA was extracted by the freeze-grinding, SDS-based methods (Zhou et al., 1996) and was purified using a low melting agarose gel followed by phenol extraction. DNA concentration and quality was determined with NanoDrop ND1000. $5 \mu \mathrm{g}$ purified DNA was partially digested with $10 \mathrm{U}$ Sau3AI (Takara) in a $100 \mu \mathrm{L}$ reaction at $37^{\circ} \mathrm{C}$ for $2 \mathrm{~h}$. Digested DNA with a size range of $1 \mathrm{~kb}-3 \mathrm{~kb}$ was selected by agarose gel electrophoresis and extracted from agarose gel using QIAquick Gel Extraction Kit (Qiagen 28704). Recovered DNA was ligated with BamHI digested pUC19 vector using the following protocol: $1 \mu \mathrm{L} 10 \times$ Ligation buffer,
80 ng partially digested DNA, 20 ng BamHI cut pUC19 vector and $0.5 \mu \mathrm{L}$ NEB T4 ligase (M0202) in a $10 \mu \mathrm{L}$ ligation reaction, followed by incubation at $16{ }^{\circ} \mathrm{C}$ for $16 \mathrm{~h}$ and heat inactivation at $65^{\circ} \mathrm{C}$ for $20 \mathrm{~min}$. $2 \mu \mathrm{L}$ fresh ligation product was transformed by electroporation into $50 \mu \mathrm{L}$ prepared electro-competent Escherichia coli DH5 $\alpha$ cells (Sambrook and Russell, 2000). After transformation using $2500 \mathrm{~V}$ for a $2 \mathrm{~mm}$ electroporation cuvette, cells were recovered with $1 \mathrm{~mL}$ SOC medium in a shaker at $37^{\circ} \mathrm{C}, 200 \mathrm{rpm}$ for $1 \mathrm{~h}$. For each sample, recovered cells were combined as a library after all the ligation products were transformed.

Libraries were titered by plating $1 \mu \mathrm{L}$ and $0.1 \mu \mathrm{L}$ recovered cells onto LB agar plates containing $100 \mu \mathrm{g} \mathrm{mL}-1$ ampicillin followed by incubation at $37{ }^{\circ} \mathrm{C}$ for $16 \mathrm{~h}$. The LB agar plates were spread with $40 \mu \mathrm{L}$ $2 \%$ X-gal and $7 \mu \mathrm{L} 20 \%$ IPTG before titering. After incubation, white colonies were selected for amplifying the insert using M13 primers flanking the BamHI site of the pUC19 vector. For each library, average insert size was calculated by PCR amplifying 15 random chosen inserts using M13 primers and agarose gel electrophoresis. Total library size was determined by multiplying average insert size with the number of colony forming unit (cfu). The rest of recovered cells were grown in $30 \mathrm{~mL}$ LB broth containing $100 \mu \mathrm{g} \mathrm{mL}{ }^{-1}$ ampicillin at $37^{\circ} \mathrm{C} 200 \mathrm{rpm}$ for $6 \mathrm{~h}$, cells were collected by centrifugation at $5000 \mathrm{rpm}$ for $15 \mathrm{~min}$ and re-suspended in $10 \mathrm{~mL}$ LB containing $100 \mu \mathrm{g} \mathrm{mL}^{-1}$ ampicillin, which were frozen in liquid nitrogen with $15 \%$ glycerol and stored in $-80^{\circ} \mathrm{C}$.

\subsection{Screening of antibiotic resistant clones}

For each library, $2 \mathrm{~mL}$ frozen stock was grown in $20 \mathrm{~mL}$ LB with $100 \mu \mathrm{g} \mathrm{mL}-1$ ampicillin at $37^{\circ} \mathrm{C}$ for $6 \mathrm{~h}$. $100 \mu \mathrm{L}$ amplified library was spread on LB agar plates containing $100 \mu \mathrm{gL}^{-1}$ ampicillin and one of the antibiotics listed in Table S1 and incubated at $37{ }^{\circ} \mathrm{C}(16 \mathrm{~h})$, $30{ }^{\circ} \mathrm{C}(2 \mathrm{~d})$ and $25^{\circ} \mathrm{C}(4 \mathrm{~d})$, with 3 replicates for each temperature. Resistant clones were transferred to fresh LB agar plates containing $100 \mu \mathrm{g} \mathrm{mL}^{-1}$ ampicillin and relevant antibiotics of which resistance had been selected. Insert of each clone was amplified with M13 primers. Insert size was determined by agarose gel electrophoresis. Amplified inserts with identical size were digested with MspI. Inserts with different RFLP pattern and different insert size were picked and sequenced using Sanger sequencing at BGI Shenzhen (BGI, Shenzhen, China). Picked clones were grown in LB medium containing $100 \mu \mathrm{gL}^{-1}$ ampicillin and relevant antibiotic to verify resistance before sequencing.

\subsection{Identification of antibiotic resistance genes}

Open reading frames (ORFs) of full length inserts were identified using ORFfinder (http://www.ncbi.nlm.nih.gov/projects/gorf/). Identified ORFs were compared to a non-redundant protein database using blastp and non-redundant nucleotide database using tblastx. The closest hit for each ORF was collected with blastp and phylogenetic analysis for all genes was performed categorized by the antibiotic class resistant phenotype. The amino acid sequences were downloaded and aligned using ClustalW methods, phylogenetic trees were constructed by the neighbor-joining method and bootstrap analysis (1000 replicates) was performed with MEGA 5.05 software package (Tamura et al., 2011).

\section{Results and discussion}

\subsection{Soil metagenomic library construction and antibiotic resistant clone isolation}

Four metagenomic libraries were constructed using DNA extracted from different agricultural soils: one from a shrimp pond sediment (YZC library), one from a field soil grown with A. fistulosum L (DC library) and two from paddy fields in Tianjin (QG and WG library). Each library contained about $2 \times 10^{5}$ recombinant clones with average 
insert size of $2 \mathrm{~kb}$. Thus each library contained about $400 \mathrm{Mb}$ DNA extracted from the soil, representing approximately 100 bacterial genome (assuming an average bacterial genome size of $4 \mathrm{Mb}$ ).

The constructed metagenomic libraries were subjected to antibiotic resistant clone isolation using each of 13 antibiotics listed in Table S1. A total of 45 unique clones were identified conferring resistance to the following antibiotics: tetracycline (TC) minocycline and tetracycline; aminoglycoside streptomycin, gentamicin, kanamycin and amikacin; chloramphenicol and rifampicin. The resistant phenotypes of selected clones were validated by checking their ability to grow on relevant antibiotics containing LB medium, followed by sequence analysis for the characterization of antibiotic resistant genes. The identified ARGs include aminoglycoside acetyltransferase, aminoglycoside 6adenyltransferase, ADP-ribosyl transferase, ribosome protection protein, transporters and other antibiotic resistant determinants. It is likely that the constructed metagenomic libraries could contain much more ARGs than that identified in this work due to the limitation of heterologous expression in the E. coli host. Given the considerable biodiversity of soil microbial communities, expanding the metagenomic library size could yield tens or hundreds more ARGs.

For all the putative resistant genes, the similarity with the closest protein in GenBank ranged from $26 \%$ to $92 \%$, with only a minority of $2 \%$ of identified resistant genes had high similarity (>90\% at amino acid level) to previous known genes, and $67 \%$ of identified resistant genes had low similarity less than $60 \%$ at amino acid level, which may indicate the novelty of these genes (Fig. 1). Most of the ARGs failed to identify genes with significant matches when using nucleotide blast search of NCBI nr database, which suggest that functional screening could discover genes whose function might not be obvious from their sequence.

Thirty one clones were derived from DC library, representing about $69 \%$ of the total resistant clones (Fig. 2). The DC library was constructed using DNA from field soil grown with A. fistulosum L., which was fertilized with pig manure. Antibiotics are commonly used in swine farm for disease treatment and growth promotion, which could lead to high concentration of antibiotics in pig manure (Qiao et al., 2012) and subsequently increase the antibiotic concentration in soil amended with pig manure. Our recent study revealed strikingly diverse and abundant ARGs in Chinese swine farms (Zhu et al., 2013), the results in this study provided another piece of evidence that antibiotic resistance in the environment could be affected directly by the release of antibioticresistant bacteria and antibiotic residues for pig manure.

\subsection{Rifampicin resistance}

Four clones were identified to be resistant to rifampicin, three from WG library and one from YZC library, the size of inserts was between
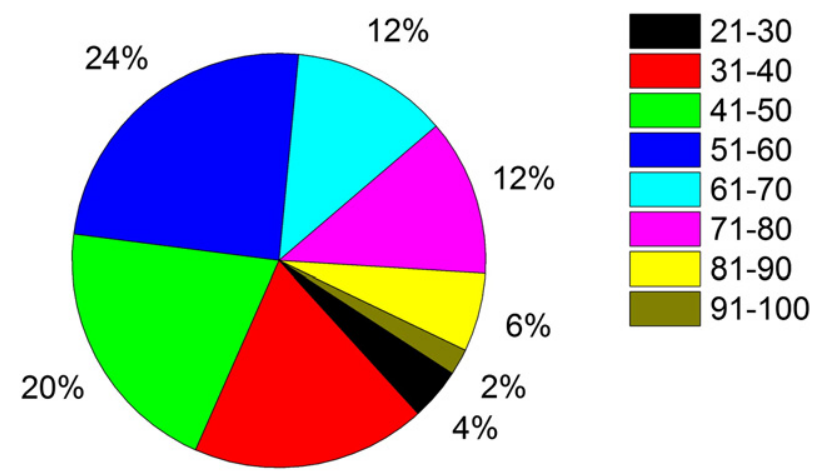

$18 \%$

Fig. 1. Amino acid similarity distribution for antibiotic resistant genes identified in this study. Amino acid similarity was obtained by comparing the amino acid sequence of identified antibiotic resistant genes in this study to that of the closest match in GenBank database. Legend indicates the percentage of sequence identity at amino acid level.

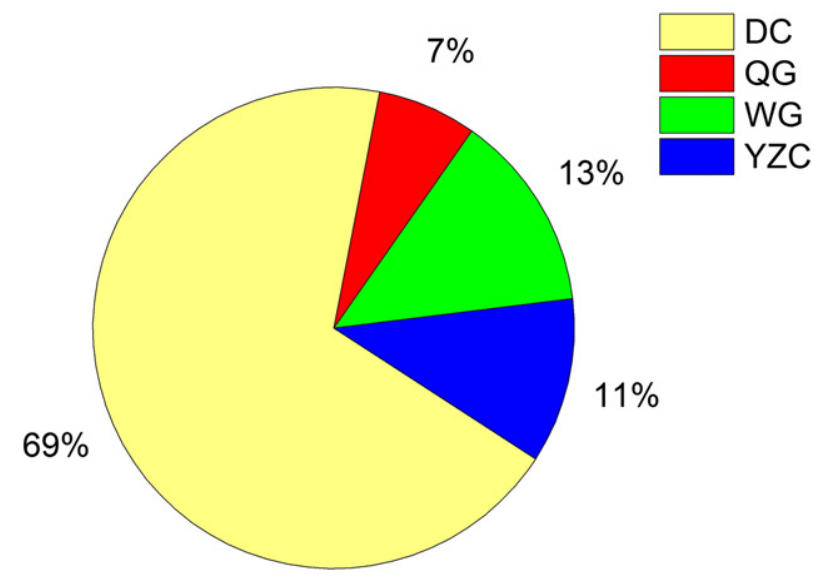

Fig. 2. Library distribution of antibiotic resistant clones. Four metagenomic libraries were constructed using DNA extracted from different agricultural soils: one from a shrimp pond sediment (YZC), one from a field soil grown with Allium fistulosum L (DC) and two from paddy fields in Tianjin (QG and WG).

1 and $4 \mathrm{~kb}$. Putative ORFs responsible for rifampicin resistant were identified, and the similarity of amino acid sequences of rifampicin resistance genes ranged from $35 \%$ to $75 \%$ to their most similar proteins (Table S2, Fig. S1). ORF WGRif3028-2 encoded a rifampin ADP-ribosyl transferase, suggesting that the conferred resistance is due to ribosylation of rifampicin. ORF WGRif3063-1 encoded a transporterlike protein. Although ORF WGRif3065 encoded a protein similar to a hypothetical protein, tblastx search database showed that it was related to a putative efflux RND transporter protein. Three ORFs were identified in clone YZCRif3706, including 1 closely related to DEAD/DEAH box helicase and 2 transporter protein homologues, sub-cloning of each ORF to an E. coli host or transposon mutagenesis should be carried out to test the ability of individual gene to confer resistance to rifampicin.

\subsection{Chloramphenicol resistance}

A total of 11 ORFs predicted to confer chloramphenicol resistance were identified from 11 recombinant clones (insert size $0.8-2 \mathrm{~kb}$ ). Most of the chloramphenicol resistant clones were derived from the DC library, except one from the WG library. These ORFs shared an amino acid identity between $32 \%$ and $89 \%$ with their closest genes in GenBank (Table S3, Fig. S2). Annotation of chloramphenicol resistant genes revealed a number of mechanisms which enable them to mitigate the inhibitory effect of chloramphenicol. ORFs DCChl2502 and DCChl2509 encoded proteins belong to the multidrug ABC transporter or the RND family efflux transporter, sharing a similarity of $70 \%$ to $48 \%$ with their closest protein, respectively. Phylogenetic analysis of the amino acid sequence of DCChl2502 and DCChl2509 revealed very low similarity with any of the known chloramphenicol/florfenicol exporter genes (Fig. 3). DCChl2503, DCChl2510, DCChl2512 and DCChl2516-2 encoded proteins are involved in ribosome protection, recycling and biogenesis, which could weaken the bacteriostatic effect of chloramphenicol, resulting in the resistance to chloramphenicol (Dittrich and Schrempf, 1992; Granneman et al., 2006; Iwanaga et al., 2004).

A serine/threonine-protein kinase like protein was encoded by DCChl3005 and shared a very low similarity (32\%) with the closest protein at amino acid level. Over-expression of this gene may lead to increased generation of persistent cells which could survive antibiotic treatment probably by entering into a dormant state and exhibit multidrug resistance (Correia et al., 2006). WGChl2502-1 encoded a PhoH family protein, which is important to starvation- and stationaryphase-induced resistance to membrane-permeabilizing antimicrobial agents (McLeod and Spector, 1996). The function of DCChl2506 encoded ATP phosphoribosyltransferase like protein, DCChl2508 encoded allantoinase like protein and DCChl2515 encoded alpha-N- 


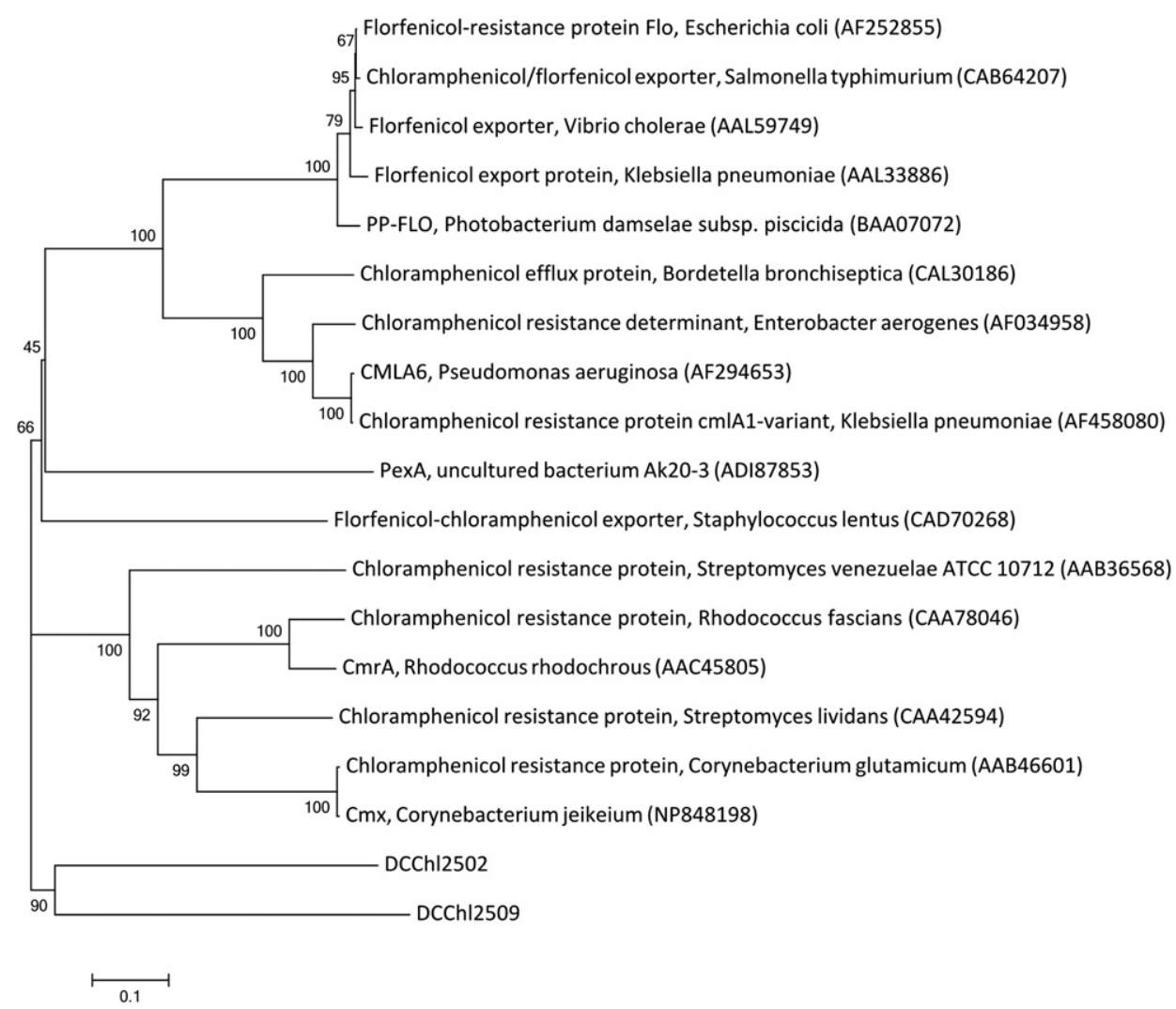

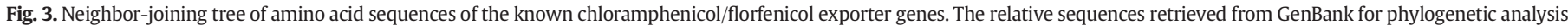
were presented with gene products, followed by genera, species and GenBank accession numbers.

arabinofuranosidase like protein conferring chloramphenicol resistance remains unknown. The most common mechanism of chloramphenicol resistance is chloramphenicol acetyltransferases (CATs) mediated by acetylation, resulting in the inactivation of chloramphenicol (Schwarz et al., 2004), however, resistance gene encoding CATs like protein were not detected in this study.

\subsection{Aminoglycoside resistance}

We identified 23 ARGs from 23 clones conferring resistance to aminoglycoside: 2 conferring resistance to amikacin, 2 to gentamycin, 1 to streptomycin and 18 to kanamycin, with amino acid similarity between $26 \%$ and $92 \%$ to their closest proteins in GenBank (Table S4). Most of the aminoglycoside resistance genes were derived from DC library, except 2 from QG library and WG library respectively. Enzymes responsible for inactivation of aminoglycosides were classified into three major classes according to the type modification: AACs (acetyltransferases), ANTs (nucleotidyltransferases or adenyltransferases), and APHs (phosphotransferases) (Ramirez and Tolmasky, 2010; Wright, 1999). Five AAC like genes (DCGen3701, DCKan30A20-3, DCKan30A30-2, WGKan37B65 and WGKan37B73-2) and one ANT like gene (QGStr371) were identified in this study (Table S4). Phylogenetic analysis of the amino acid sequences of identified genes with reported AACs and ANTs showed that DCGen3701, sharing a low similarity of 38\% with the closest match in GenBank database, was related to an AAC(3) cluster proteins, while it was separated from the other twelve $A A C(3)$ proteins, indicating that it could be a novel AAC(3) cluster gene. WGKan37B65, WGKan37B73-2 and DCKan30A30-2 fell within the cluster of AAC $\left(6^{\prime}\right)$ enzymes which are by far the most common (Ramirez and Tolmasky, 2010), while DCKan30A30-2 was distant from other $A A C\left(6^{\prime}\right)$ forming a separate branch in the $\mathrm{AAC}\left(6^{\prime}\right)$ cluster, suggesting that it may be a new AAC $\left(6^{\prime}\right)$ gene. DCKan30A20-3 was found to be related to an $\mathrm{AAC}\left(2^{\prime}\right)$ gene and form a separate cluster (Fig. 4). QGStr37-1 shared a high similarity of $70 \%$ at amino acid level with an aminoglycoside 6adenyltransferase from Sphingobacterium spiritivorum (Table S4), they group together and form a new cluster distinctive from ANT(6)-Ia proteins (Fig. 5).

Other putative inactivation enzymes were also identified in this study, including 2 fumarylacetoacetate (FAA) hydrolase like proteins (DCAmi3001-1, DCKan30A25-2) and 1 HAD family hydrolase like protein (DCKan30A12). Interestingly, we found DCKan30A10, which encoded an erythromycin esterase like protein, exhibited resistance to kanamycin, while further experiments showed that clone DCKan30A10 could not grow in erythromycin containing LB medium.

Ribosome alteration including methylation of $16 \mathrm{~S}$ rRNA, mutation of 16S rRNA or ribosomal proteins would lead to aminoglycoside resistance (Doi and Arakawa, 2007; Galimand et al., 2005). Methylase like protein encoding genes were also detected conferring resistance to kanamycin, including ORFs DCKan30A22-2, DCKan30A43-2 and QGKan37-1.

Reduced uptake, decreased permeability or export by active efflux pump is another major mechanisms involved in aminoglycoside resistance (Ramirez and Tolmasky, 2010). DCGen3002-1 encoded a protein similar to phosphomannomutase and DCKan30A29 encoded a protein similar to nucleotide sugar dehydrogenase, both of which are responsible for alginate polymerization and the resultant alginate layer would cause a mucoid phenotype and provide a protective barrier against host immune defenses and antibiotics (May et al., 1991; Naught et al., 2002). DCKan30A03 encoded 2-oxoglutarate dehydrogenase is involved in glutamine utilization and could reduce cell wall hydrophobicity, which has previously been reported to be important for multiple antibiotic resistance (Wolff et al., 2009). Efflux pump mediated resistance was found in $\mathrm{ABC}$ transporter like protein encoded DCKan30A31-2.

Other mechanisms for aminoglycoside resistance were also found in this study, such as attenuation of oxidative stress caused by aminoglycoside (DCAmi3003-2, DCKan30A27), regulation of marRAB multiple 


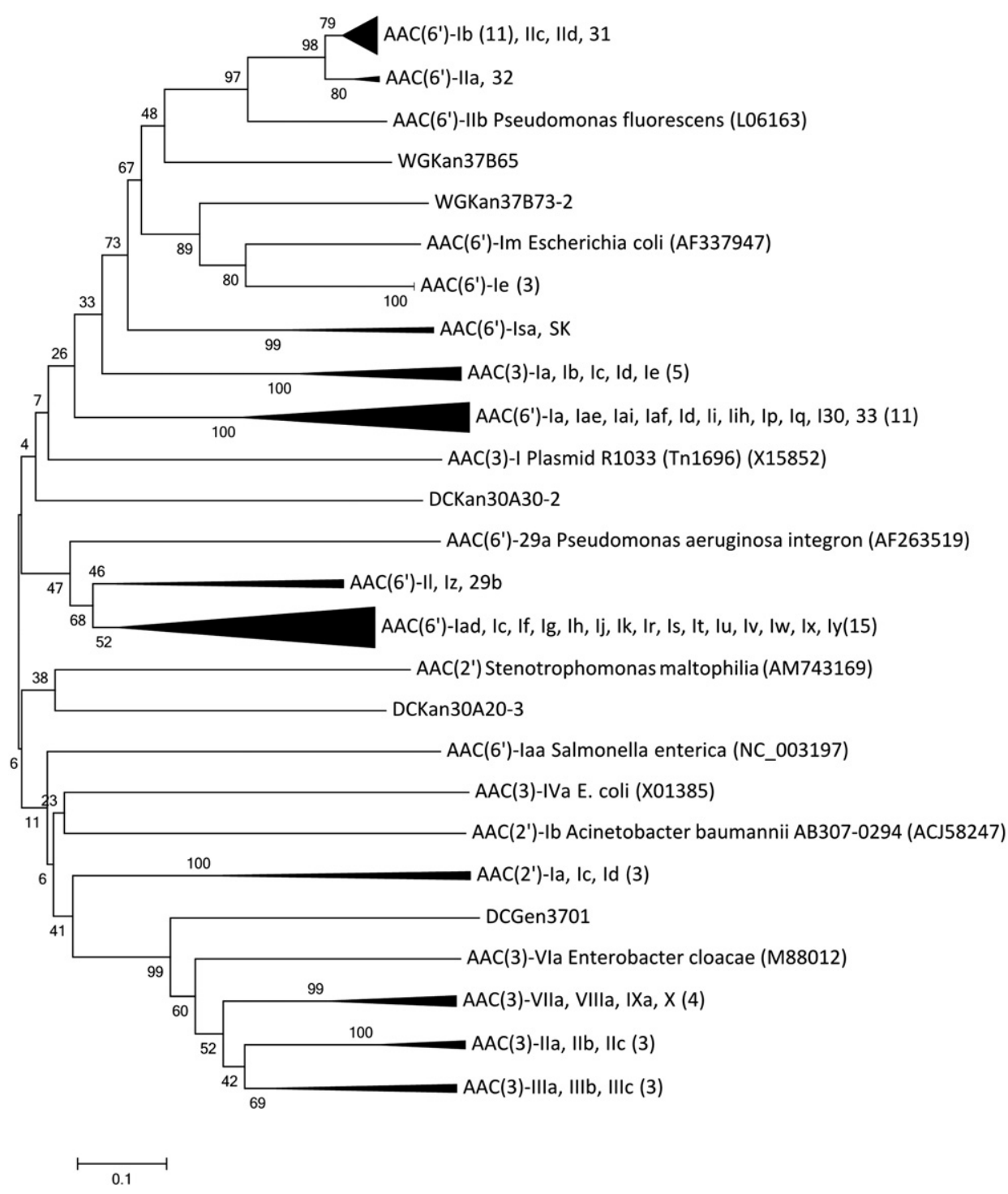

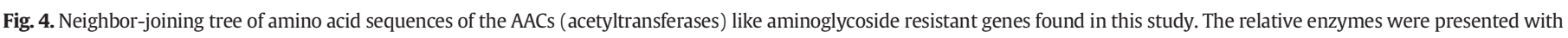

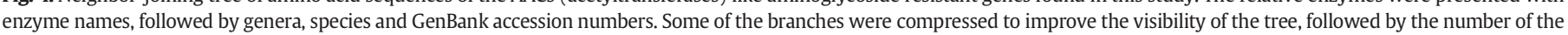
grouped sequences in parentheses.

antibiotic resistance operon by DCKan30A19-2 encoded transketolaselike protein (Domain et al., 2007) and DCKan30B13-2 encoded tRNA synthetase mediated resistance (Struble and Gill, 2009). Although previous reports showed that UvrA homolog confers resistance to nogalamycin and daunorubicin (Goosen and Moolenaar, 2008), the function of DCKan30B21 encoded UV damage endonuclease in the kanamycin resistance remains unknown. Three ORFs were identified in clone DCKan30A08 and were closely related to aminotransferase class-III, Isoprenylcysteine carboxyl methyltransferase and glyoxalase/ bleomycin resistance protein/dioxygenase, respectively. The ORF in clone DCKan30A08 responsible for kanamycin resistance remained to be determined.

\subsection{Tetracycline resistance}

A total of seven clones were identified: five conferring resistance to tetracycline and two to minocycline. Two of the TC resistant clones were derived from DC library, one from QG library, and four from YZC library, respectively (Table S5). Efflux of TCs out of cells and ribosome protection are the most widespread mechanisms in TC resistance, and most of their genes are acquired via transferable plasmids and/or transposons (Thaker et al., 2010). TC efflux pumps like genes were identified in this study, they were DCTet3003-1, YZCMin2541 and YZCTet3735. Phylogenetic analysis indicated that DCTet3003-1 and YZCTet3735 were related to tetV, while YZCMin2541 was related to otrC (Fig. S3).

Tetracycline inactivation enzymes were relatively few with only three genes, tet(X), tet(34) and tet(37), were reported conferring tetracycline resistance due to alteration of tetracycline (Roberts, 2005). While, in this study, QGMin2501 was identified encoding an amidohydrolase like protein which could hydrolyse of methylamino of minocycline, resulting in inactivation of minocycline. The serine/threonine protein kinase mediated resistance, as mentioned before, was also observed in DCTet3002 (nucleotide sequence identical to DCChl3005), YZCTet3012 and YZCTet3064-1.

ARGs conferring resistance to beta-lactams (Cefotaxime), fluoroquinolone (Ciprofloxacin), macrolides (Erythromycin), trimethoprim and nitrofurantoin were not detected in this study, which may be due to the cloning bias and selective heterogeneous expression of environmental DNA in E. coli (Forns et al., 1997). Although functional screening of metagenomic libraries is a powerful approach in the study of ARGs, it has the disadvantage that the gene expression is necessary for the selection, cryptic genes or gene unable to be expressed in the recipient strain could be missed during the functional screening. Given that the quantity and diversity of microbes in soil, the constructed metagenomic library 


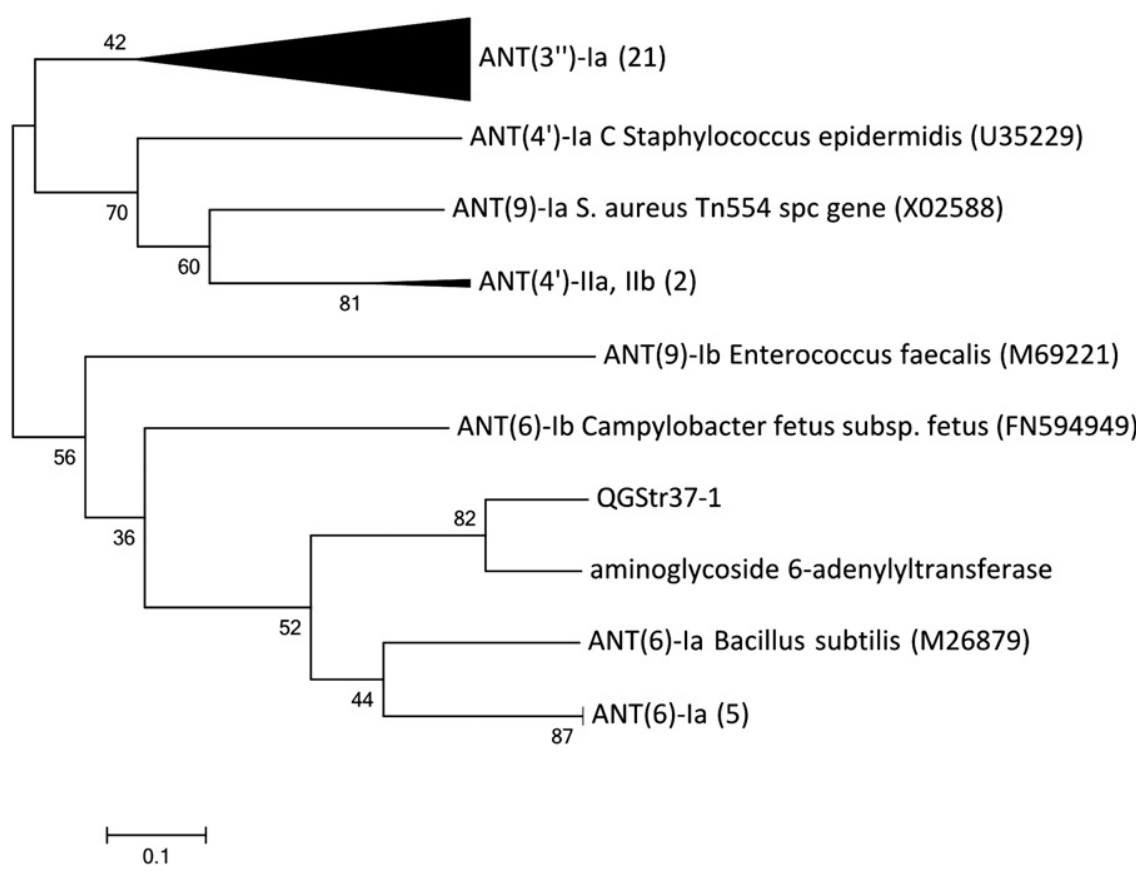

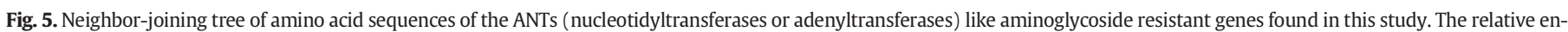

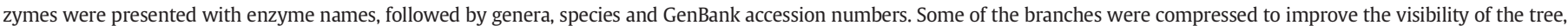
followed by the number of the grouped sequences in parentheses.

represents only a small fraction of microbial community in soil, and thus, the true size of the soil ARGs reservoir could be much larger (Monier et al., 2011). Some of the ARGs were closely linked with a number of widespread mobile genetic elements (MGEs), including plasmids, transposons and integrons, which were involved in horizontal transfer of ARGs among environmental bacteria (Fluit and Schmitz, 1999; Fricke et al., 2009; Li et al., 2012; Zhang et al., 2011). These ARGs could be missed when screening a microbial chromosomal DNA derived metagenomic library.

Our results showed that most of the identified ARGs had low similarity at amino acid level with the closest protein in GenBank, suggesting that soil ARGs might remain largely uncovered. Antibiotics in the natural environment exist at a concentration much lower than the therapeutic dose, and play multifaceted roles in nature, including inter-species competition, signal communication, host-parasite interaction, virulence modulation and quorum sensing (Sengupta et al., 2013). Therefore, unlike clinical environment, in which the main function of ARGs is to confer protection against the lethal concentrations of antibiotics, ARGs harbored in the natural environment also serve for response to antibiotic signaling (Aminov, 2009) and confer resistance not only to antibiotics but also to a number of structurally unrelated compounds (Sengupta et al., 2013). This could be one reason for the disparity between soil ARGs and clinical resistance. Future work should be carried out ascertain the effect and mechanisms of novel ARGs.

\section{Conclusion}

This study is the first effort to characterization antibiotic resistome for multiple antibiotics from agricultural soil in China using functional metagenomic method. We highlight the advantage of functional metagenomic technology in discovering novel ARGs and resistant mechanisms rather than identifying genes on the basis of sequences. We identified a variety of ARGs conferring resistance to rifampicin, chloramphenicol, aminoglycoside and tetracycline, through almost all known mechanisms underlying relative antibiotic resistant phenotype. The novelty and diversity of identified ARGs suggest that antibiotic resistance in soil remains unexplored and could potentially threat human health if they transfer to pathogens. The identified novel ARGs and resistant mechanisms will extend our knowledge and understanding of the ecology of microbial antibiotic resistance in different environments, and will shed new insights on the emergence of ARGs in natural environment.

\section{Acknowledgments}

This work was supported by the Knowledge Innovation Program of the Chinese Academy of Sciences (KZCX2-EW-QN410 and KZCX2-YWJC402) and the National Natural Science Foundation of China (21210008, 41090282).

\section{Appendix A. Supplementary data}

Supplementary data to this article can be found online at http://dx. doi.org/10.1016/j.envint.2013.12.010.

\section{References}

Allen HK, Moe LA, Rodbumrer J, Gaarder A, Handelsman J. Functional metagenomics reveals diverse beta-lactamases in a remote Alaskan soil. ISME J 2009;3:243-51.

Aminov RI. The role of antibiotics and antibiotic resistance in nature. Environ Microbiol 2009;11:2970-88.

Bush K, Courvalin P, Dantas G, Davies J, Eisenstein B, Huovinen P, et al. Tackling antibiotic resistance. Nat Rev Microbiol 2011:9:894-6.

Correia FF, D'Onofrio A, Rejtar T, Li L, Karger BL, Makarova K, et al. Kinase activity of overexpressed HipA is required for growth arrest and multidrug tolerance in Escherichia coli. J Bacteriol 2006;188:8360-7.

D'Costa VM, McGrann KM, Hughes DW, Wright GD. Sampling the antibiotic resistome. Science 2006;311:374-7.

Dittrich W, Schrempf H. The unstable tetracycline resistance gene of Streptomyces lividans 1326 encodes a putative protein with similarities to translational elongation factors and Tet(M) and Tet(O) proteins. Antimicrob Agents Chemother 1992;36:1119-24.

Doi Y, Arakawa Y. 16S ribosomal RNA methylation: emerging resistance mechanism against aminoglycosides. Clin Infect Dis 2007;45:88-94.

Domain F, Bina XR, Levy SB. Transketolase A, an enzyme in central metabolism, derepresses the marRAB multiple antibiotic resistance operon of Escherichia coli by interaction with MarR. Mol Microbiol 2007;66:383-94.

Donato JJ, Moe LA, Converse BJ, Smart KD, Berklein FC, McManus PS, et al. Metagenomic analysis of apple orchard soil reveals antibiotic resistance genes encoding predicted bifunctional proteins. Appl Environ Microbiol 2010;76:4396-401.

Fluit AC, Schmitz FJ. Class 1 integrons, gene cassettes, mobility, and epidemiology. Eur J Clin Microbiol 1999;18:761-70. 
Forns X, Bukh J, Purcell RH, Emerson SU. How Escherichia coli can bias the results of molecular cloning: preferential selection of defective genomes of hepatitis $\mathrm{C}$ virus during the cloning procedure. Proc Natl Acad Sci 1997;94:13909-14.

Fricke WF, Welch TJ, McDermott PF, Mammel MK, LeClerc JE, White DG, et al. Comparative genomics of the IncA/C multidrug resistance plasmid family. J Bacteriol 2009;191:4750-7.

Galimand M, Lambert T, Courvalin P. Emergence and dissemination of a new mechanism of resistance to aminoglycosides in Gram-negative bacteria: 16S rRNA methylation. Euro surveillance: bulletin europeen sur les maladies transmissibles = European communicable disease bulletin; 2005 [10:E050127 050122].

Gaze WH, Zhang L, Abdouslam NA, Hawkey PM, Calvo-Bado L, Royle J, et al. Impacts of anthropogenic activity on the ecology of class 1 integrons and integron-associated genes in the environment. ISME J 2011;5:1253-61.

Ghosh S, LaPara TM. The effects of subtherapeutic antibiotic use in farm animals on the proliferation and persistence of antibiotic resistance among soil bacteria. ISME J 2007;1:191-203.

Goosen N, Moolenaar GF. Repair of UV damage in bacteria. DNA Repair (Amst) 2008;7: 353-79.

Granneman S, Bernstein KA, Bleichert F, Baserga SJ. Comprehensive mutational analysis of yeast DEXD/H box RNA helicases required for small ribosomal subunit synthesis. Mol Cell Biol 2006;26:1183-94.

Heuer H, Schmitt H, Smalla K. Antibiotic resistance gene spread due to manure application on agricultural fields. Curr Opin Microbiol 2011;14:236-43.

Iwanaga M, Toma C, Miyazato T, Insisiengmay S, Nakasone N, Ehara M. Antibiotic resistance conferred by a class I integron and SXT constin in Vibrio cholerae 01 strains isolated in Laos. Antimicrob Agents Chemother 2004;48:2364-9.

Kazimierczak KA, Scott KP, Kelly D, Aminov RI. Tetracycline resistome of the organic pig gut. Appl Environ Microbiol 2009;75:1717-22.

Knapp CW, Dolfing J, Ehlert PAI, Graham DW. Evidence of increasing antibiotic resistance gene abundances in archived soils since 1940. Environ Sci Technol 2010;44:580-7.

Lang KS, Anderson JM, Schwarz S, Williamson L, Handelsman J, Singer RS. Novel florfenicol and chloramphenicol resistance gene discovered in Alaskan soil by using functional metagenomics. Appl Environ Microbiol 2010;76:5321-6.

Li J, Wang T, Shao B, Shen J, Wang S, Wu Y. Plasmid-mediated quinolone resistance genes and antibiotic residues in wastewater and soil adjacent to swine feedlots: potential transfer to agricultural lands. Environ Health Perspect 2012;120:1144-9.

Looft T, Johnson TA, Allen HK, Bayles DO, Alt DP, Stedtfeld RD, et al. In-feed antibiotic effects on the swine intestinal microbiome. Proc Natl Acad Sci U S A 2012;109: 1691-6.

Luo Y, Mao D, Rysz M, Zhou Q, Zhang H, Xu L, et al. Trends in antibiotic resistance genes occurrence in the Haihe River, China. Environ Sci Technol 2010;44:7220-5.

May TB, Shinabarger D, Maharaj R, Kato J, Chu L, DeVault JD, et al. Alginate synthesis by Pseudomonas aeruginosa: a key pathogenic factor in chronic pulmonary infections of cystic fibrosis patients. Clin Microbiol Rev 1991;4:191-206.

McGarvey KM, Queitsch K, Fields S. Wide variation in antibiotic resistance proteins identified by functional metagenomic screening of a soil DNA library. Appl Environ Microbiol 2012:78:1708-14.

McLeod GI, Spector MP. Starvation- and stationary-phase-induced resistance to the antimicrobial peptide polymyxin B in Salmonella typhimurium is RpoS (sigma $(S))$ independent and occurs through both phoP-dependent and -independent pathways. J Bacterio 1996;178:3683-8.

Monier J-M, Demanèche S, Delmont TO, Mathieu A, Vogel TM, Simonet P. Metagenomic exploration of antibiotic resistance in soil. Curr Opin Microbiol 2011;14:229-35.
Mori T, Mizuta S, Suenaga H, Miyazaki K. Metagenomic screening for bleomycin resistance genes. Appl Environ Microbiol 2008;74:6803-5.

Naught LE, Gilbert S, Imhoff R, Snook C, Beamer L, Tipton P. Allosterism and cooperativity in Pseudomonas aeruginosa GDP-mannose dehydrogenase. Biochemistry US 2002;41: 9637-45.

Popowska M, Rzeczycka M, Miernik A, Krawczyk-Balska A, Walsh F, Duffy B. Influence of soil use on prevalence of tetracycline, streptomycin, and erythromycin resistance and associated resistance genes. Antimicrob Agents Chemother 2012:56:1434-43.

Qiao M, Chen W, Su J, Zhang B, Zhang C. Fate of tetracyclines in swine manure of three selected swine farms in China. J Environ Sci 2012;24:1047-52.

Ramirez MS, Tolmasky ME. Aminoglycoside modifying enzymes. Drug Resist Updat 2010;13:151-71.

Riesenfeld CS, Goodman RM, Handelsman J. Uncultured soil bacteria are a reservoir of new antibiotic resistance genes. Environ Microbiol 2004;6:981-9.

Roberts MC. Update on acquired tetracycline resistance genes. FEMS Microbiol Lett 2005;245:195-203.

Sambrook J, Russell D. Molecular cloning: a laboratory manual. 3rd ed. Cold Spring Harbor Laboratory Press; 2000.

Schwarz S, Kehrenberg C, Doublet B, Cloeckaert A. Molecular basis of bacterial resistance to chloramphenicol and florfenicol. FEMS Microbiol Rev 2004;28:519-42.

Sengupta S, Chattopadhyay MK, Grossart H-P. The multifaceted roles of antibiotics and antibiotic resistance in nature. Front Microbiol 2013:4.

Sommer MOA, Dantas G, Church GM. Functional characterization of the antibiotic resistance reservoir in the human microflora. Science 2009;325:1128-31.

Struble JM, Gill RT. Genome-scale identification method applied to find cryptic aminoglycoside resistance genes in Pseudomonas aeruginosa. PLoS One 2009;4:e6576.

Tamura K, Peterson D, Peterson N, Stecher G, Nei M, Kumar S. MEGA5: molecular evolutionary genetics analysis using maximum likelihood, evolutionary distance, and maximum parsimony methods. Mol Biol Evol 2011;28:2731-9.

Thaker M, Spanogiannopoulos P, Wright GD. The tetracycline resistome. Cell Mol Life Sci 2010;67:419-31.

Torres-Cortes G, Millan V, Ramirez-Saad HC, Nisa-Martinez R, Toro N, Martinez-Abarca F. Characterization of novel antibiotic resistance genes identified by functional metagenomics on soil samples. Environ Microbiol 2011;13:1101-14.

Wolff KA, Nguyen HT, Cartabuke RH, Singh A, Ogwang S, Nguyen L. Protein kinase G is required for intrinsic antibiotic resistance in mycobacteria. Antimicrob Agents Chemother 2009;53:3515-9.

Wright GD. Aminoglycoside-modifying enzymes. Curr Opin Microbiol 1999;2:499-503.

Wright GD. The antibiotic resistome: the nexus of chemical and genetic diversity. Nat Rev Microbiol 2007:5:175-86.

Wu N, Qiao M, Zhang B, Cheng WD, Zhu YG. Abundance and diversity of tetracycline resistance genes in soils adjacent to representative swine feedlots in China. Environ Sci Technol 2010;44:6933-9.

Zhang XX, Zhang T. Occurrence, abundance, and diversity of tetracycline resistance genes in 15 sewage treatment plants across China and other global locations. Environ Sci Technol 2011;45:2598-604

Zhang T, Zhang X-X, Ye L. Plasmid metagenome reveals high levels of antibiotic resistance genes and mobile genetic elements in activated sludge. PLoS One 2011;6:e26041.

Zhou J, Bruns MA, Tiedje JM. DNA recovery from soils of diverse composition. Appl Environ Microbiol 1996;62:316-22.

Zhu YG, Johnson TA, Su JQ Qiao M, Guo GX, Stedtfeld RD, et al. Diverse and abundant antibiotic resistance genes in Chinese swine farms. Proc Natl Acad Sci U S A 2013;110: $3435-40$ 